\title{
Cushing's Syndrome in a Pregnant Woman
}

\section{A R T I C L E I N F O}

\section{Article Type}

Case Report

\section{Authors}

Almasi Nasrabadi M.* $M D$

Mirarmandehi S.B. ${ }^{1} M D$,

Roostaee Z. ${ }^{1} \mathrm{PhD}$

\section{How to cite this article}

Almasi Nasrabadi M, Mirarman-

dehi S B, Roostaee Z. Cushing's

Syndrome in a Pregnant Woman

Sarem Journal of Reproductive Medicine. 2018;2(2):85-89.
*Sarem Fertility \& Infertility Research Center (SAFIR), Sarem Women's Hospital, Tehran, Iran

${ }^{1}$ Sarem Fertility \& Infertility Research Center (SAFIR), Sarem Women's Hospital, Tehran, Iran

\section{Correspondence}

Address: Sarem Women's Hospital, Basij Square, Phase 3, Ekbatan Town, Tehran, Iran. Postal Code: 1396956111

Phone: +98 (21) 44670888

Fax: +98 (21) 44670432

zrmj1394@gmail.com

\section{Article History}

Received: December 22, 2016

Accepted: May 18, 2017

ePublished: June 15, 2018

\begin{abstract}
A B S T R A C T
Patient Information Pregnancy is rare in most women with Cushing's syndrome (CS) due to the lack of ovulation. A 36-year-old woman, who had experienced cesarean section in her first pregnancy and her second pregnancy had occurred despite the Intrauterine Device (IUD), was referred to the hospital. The results of routine pregnancy tests and screening tests indicated normal general conditions. At the 12th week of pregnancy, the first manifestation of blood pressure was observed. During the pregnancy, the patient was constantly monitored and, finally, at the 34th week, she underwent cesarean section due to the severity of symptoms, cesarean section history, and delayed fetal infarction. A boy was born, weighing 1700 grams and was hospitalized in the NICU ward. Regarding the lack of control of blood pressure after pregnancy, an abdominal MRI was asked for the patient to examine the renal artery; a $3 \mathrm{~cm}$ mass was reported in her right adrenal, and a diagnosis of Cushing's syndrome was presented to her. The right laparoscopic adrenalectomy was conducted for the patient and gradually the Corton intake was stopped. After the recent procedures, all of the patient's symptoms, including hypertension, edema, proteinuria, hyperglycemia, and hyperlipidemia were resolved. Proximal muscles pain and weakness remained about 1 year after her laparoscopic adrenalectomy, and they were improved by physiotherapy.

Conclusion Secondary hypertension can occur due to Renovascular hypertension, pheochromocytoma, and Cushing's syndrome. Although Cushing's syndrome is rare in pregnancy, these cases can be accompanied by hypertension, preeclampsia, preterm labor, and fetal loss. Therefore, a more complete assessment and attention to important symptoms such as blood pressure is necessary to prevent the complications.
\end{abstract}

Keywords Cushing's Syndrome; Hypertension; Pregnancy

\section{CITATION LIN KS}

[1] Management of adrenal tumors in pregnancy [2] Cushing's syndrome in pregnancy: A case report and mini review of the literature [3] Adrenalectomy for adrenocortical adenoma causing Cushing's syndrome in pregnancy: A case report and review of literature [4] Adrenal disease in pregnancy [5] Cushing's syndrome during pregnancy secondary to adrenal adenoma [6] Cushing's syndrome in pregnancy [7] Cushing's syndrome in pregnancy: Report of a case and review of the literature [8] Pregnancy-induced Cushing's syndrome in recurrent pregnancies: Case report and literature review [9] Adrenal disorders in pregnancy [10] The medical management of Cushing's syndrome during pregnancy [11] Diagnosis and management of 253 Cases with Cushing's syndrome in Imam Khomeini Hospital [12] Cushing's syndrome in pregnancy: An overview [13] Adrenal tumours in pregnancy: Diagnostic challenge and management dilemma [14] Management of endocrine disease: Management of pregnant patients with Cushing's syndrome [15] Cushing's syndrome and pregnancy 


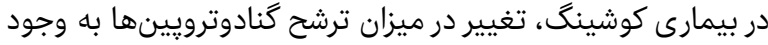

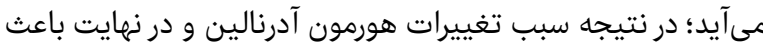

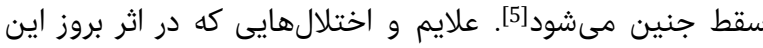

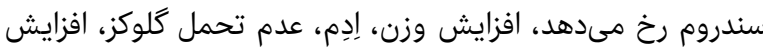

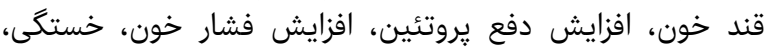

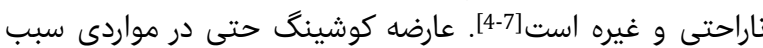

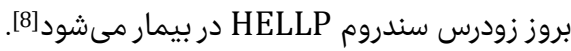
تشخيص سندروم كوشينگ در دوران باردارى بسيار مشكل استر است،

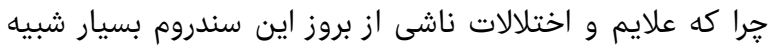

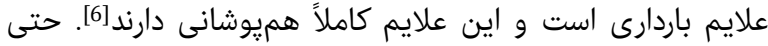

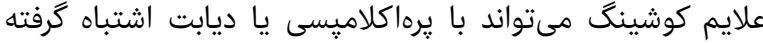

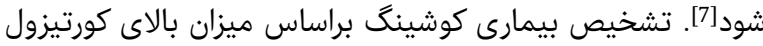

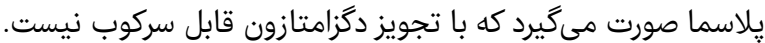

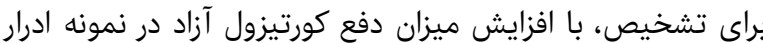

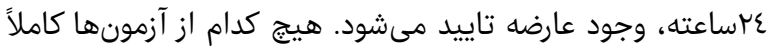

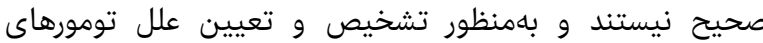

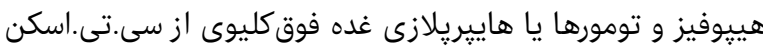

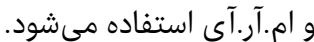

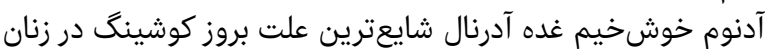

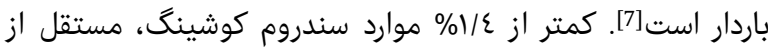

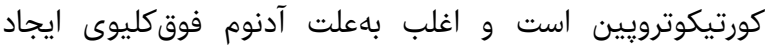

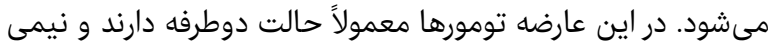

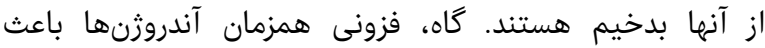

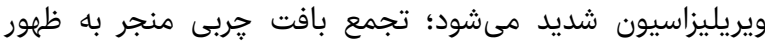

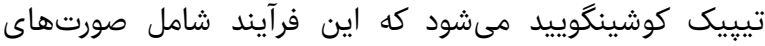

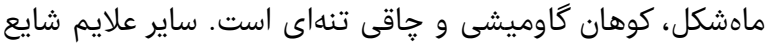

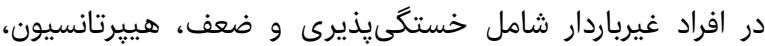

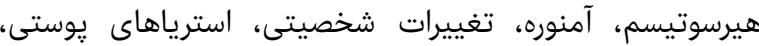

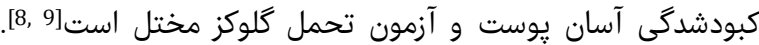

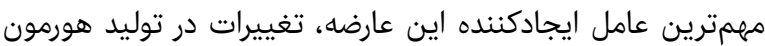

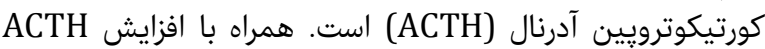

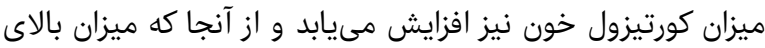

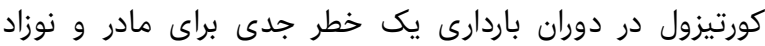

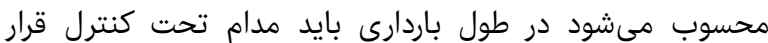

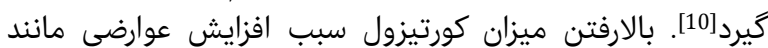

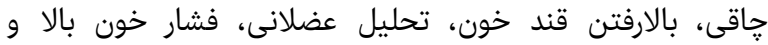

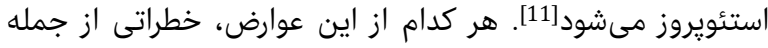

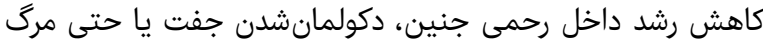
داخل رحمى جنين را مىتواند بهمئراه داشته دكان باشد.

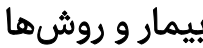

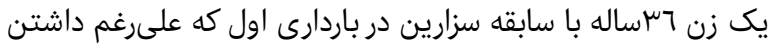

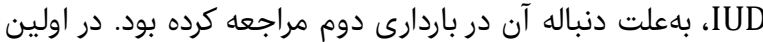

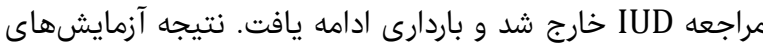

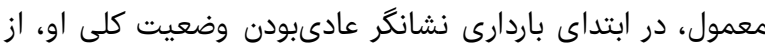

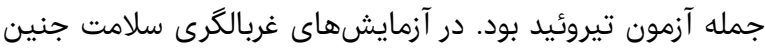

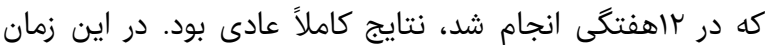

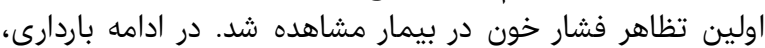

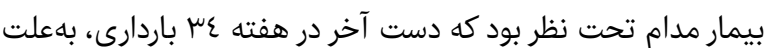

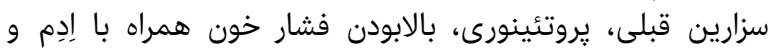

ابتلا به سندروم كوشينگ در يك خارئ خانم باردار؛

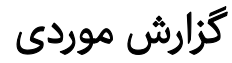

MD مريم الماسى نصرآبادى

مركز تحقيقات بارورى و نابارورى صارم، بيمارئ بارستان فوق تخصصى صارم، تهران،

ايران

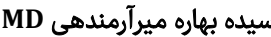

مركز تحقيقات بارورى و نابارورى صارم، بيمارستان فوق تخصصى صارم، تهران،

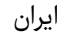

زهرا روستايى PhD

مركز تحقيقات بارورى و نابارورى صارم، بيمارستان فوق تخصصى صارم، تهران،

جكيده

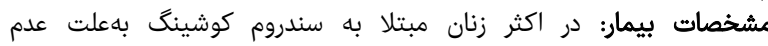

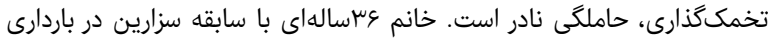

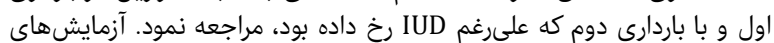

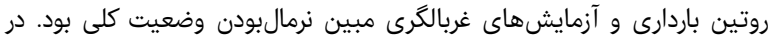

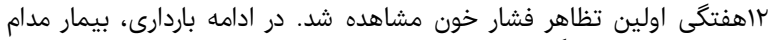

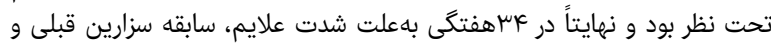

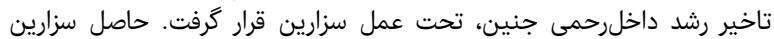

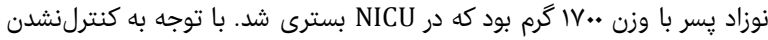

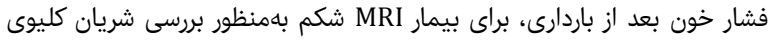

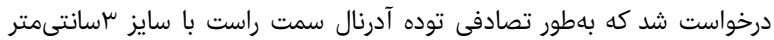

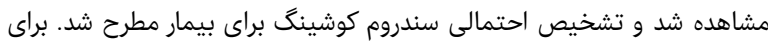

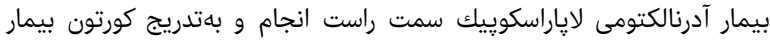

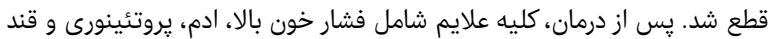

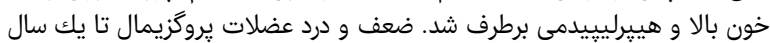

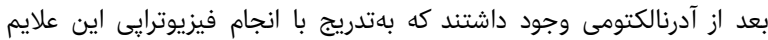

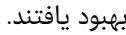

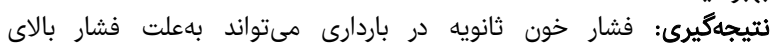

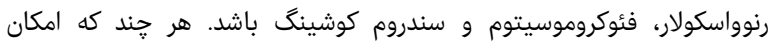

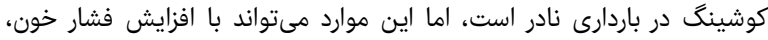

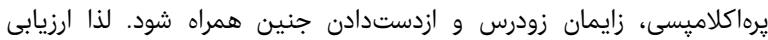

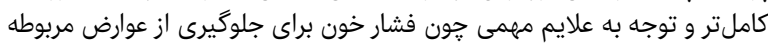

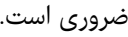

كليدوازمها: سندروم كوشينگ، فشار خون ثانويه يايدار، باردارى

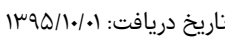

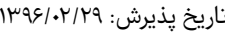

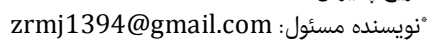

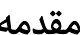

بيمارىهاى آدرنال از جمله بيمارىهاى نادر در باردارى بوده و

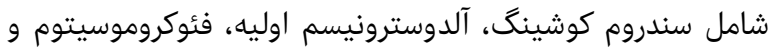

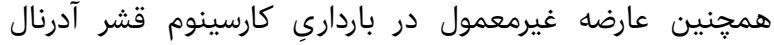

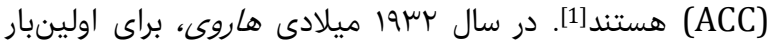

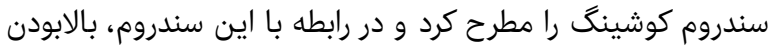

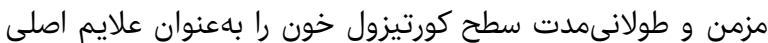

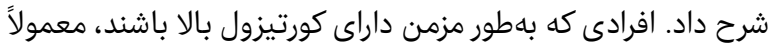

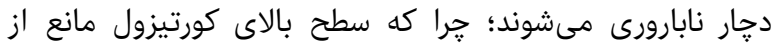

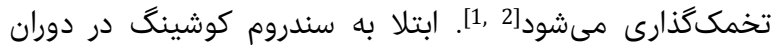

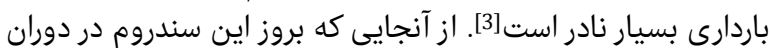

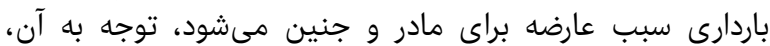

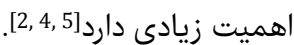




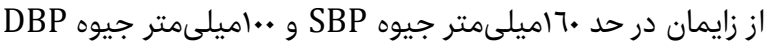

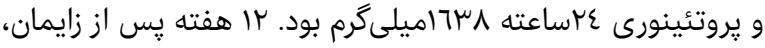

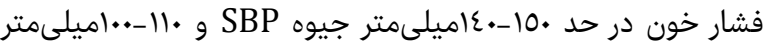

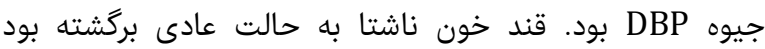
حالت ضيلى

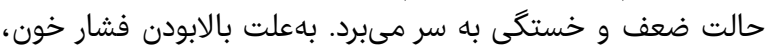

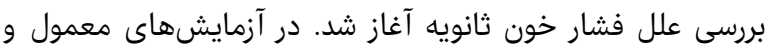

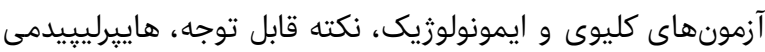

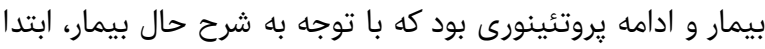

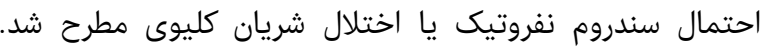

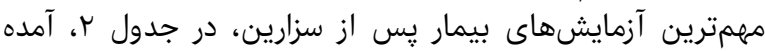

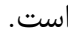

جدول () آزمايشهاى قبل از زايمان

\begin{tabular}{|c|c|c|}
\hline سن باردارى & نتيجه & آزمايشهاى قبل از زايمان \\
\hline \multirow{3}{*}{ re } & $1 \wedge \Delta$ & GCT $(\mathrm{mg} / \mathrm{dl})$ \\
\hline & $k \cdot \Delta$ & (mg/24hrs) ادرار Frساعته Pr Pr \\
\hline & $\mu / q$ & Uric acid $(\mathrm{mg} / \mathrm{dl})$ \\
\hline \multirow{5}{*}{ rq } & $r \cdot \cdot$ & يكساعته (mg/dl) يكTT \\
\hline & IEY & (mg/dl) دوساعته GTT \\
\hline & $I F \Delta$ & (mg/dl) سهساعته GTT \\
\hline & 1.94 & 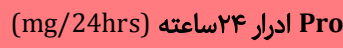 \\
\hline & $\Delta / \mathcal{F}$ & Uric acid $(\mathrm{mg} / \mathrm{dl})$ \\
\hline$\mu$. & $r / r$ & رندوم ادرار Pro/cr \\
\hline \multirow{2}{*}{ wr } & $+k$ & U/A \\
\hline & Ik. & FBS $(\mathrm{mg} / \mathrm{dl})$ \\
\hline س & $+k$ & U/A \\
\hline$\mu \mu$ & S.rQ & (mg/24hrs) ادرار Mrf (mاعته Pro \\
\hline
\end{tabular}

جدول r) آزمايشهاى بعد از زايمان

\begin{tabular}{|c|c|c|}
\hline تاريخ & نتيجه & آزمايشهاى بعد از زايمان \\
\hline آروز بعد از زايمان & KTAK & (mg/24hrs) ادرار Frاعاعته Pr \\
\hline \multirow[t]{4}{*}{ سماه پِ از زايمان } & rTV & $\mathbf{T G}(\mathrm{mg} / \mathrm{dl})$ \\
\hline & $k \cdot 1$ & CHOL $(\mathrm{mg} / \mathrm{dl})$ \\
\hline & 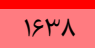 & (mg/24hrs) ادرار Pr Pr Pr (m) \\
\hline & منفى & نستهاى ترومبوفيلى \\
\hline \multirow[t]{5}{*}{ ل إعاه يس از زايمان } & $\Delta / V$ & HbA1C \\
\hline & كمتر از & АCTH $(\mathrm{Pg} / \mathrm{ml})$ \\
\hline & 19 & Cortisol 8AM (Mcg/l) \\
\hline & 99. & Free Cortisol $(\mu \mathrm{g} / 24 \mathrm{hrs})$ \\
\hline & 11 & Cortisol following medicine \\
\hline
\end{tabular}

براى بيمار ام.آر.آى شكم بهمنظور بررسى شريان كليوى درخواست

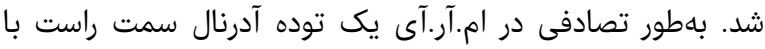

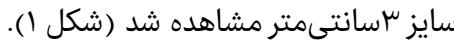

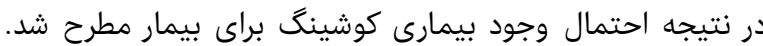

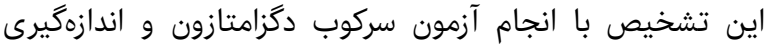

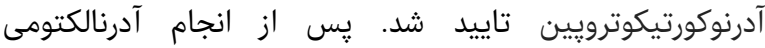

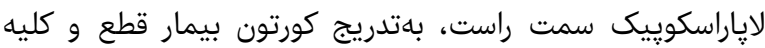

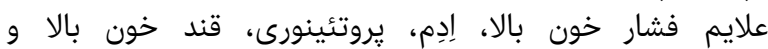

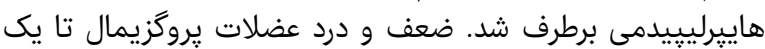

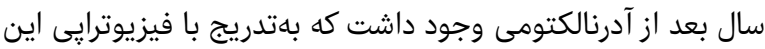

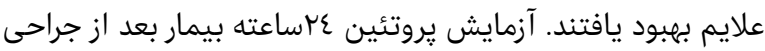

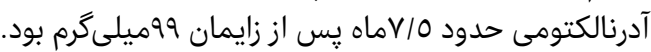

افزايش سطح قند خون و تاخير رشد داخل رحمى جنين، بيمار

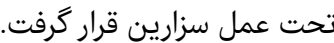
در طول باردارى، بيمار دجار فشار خون خرن بار بالا بود و بعد از باردارى نيز

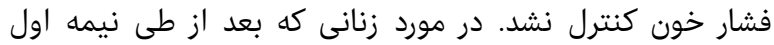

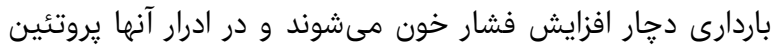

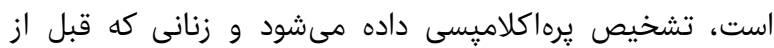

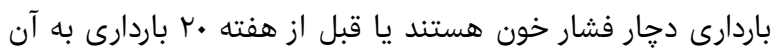

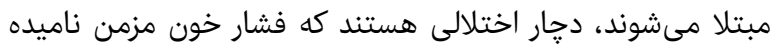

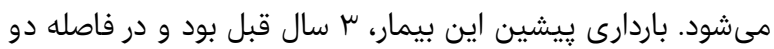

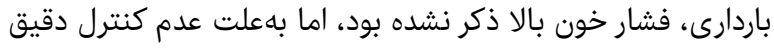

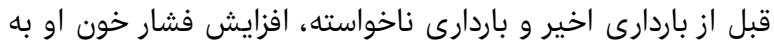

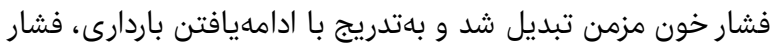

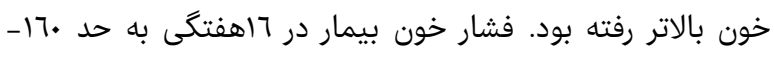

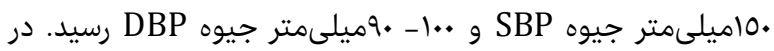

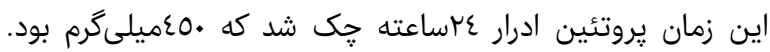

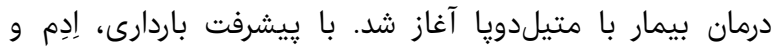

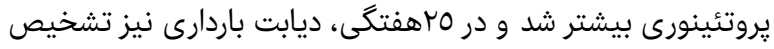

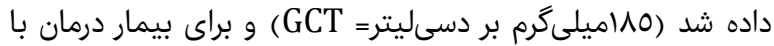

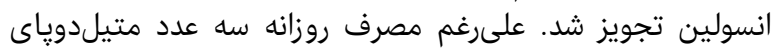

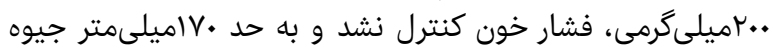

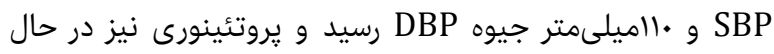

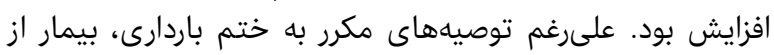

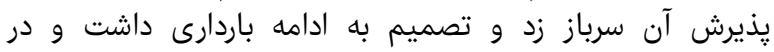

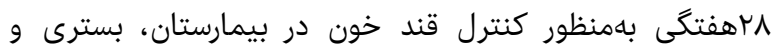

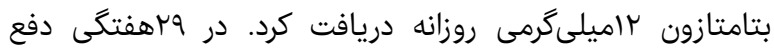

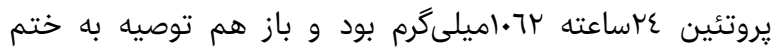

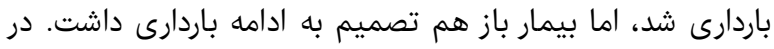

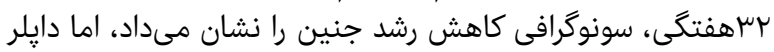

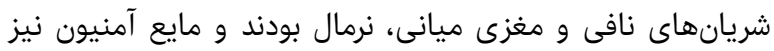

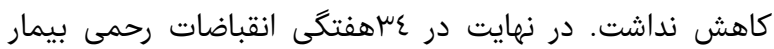

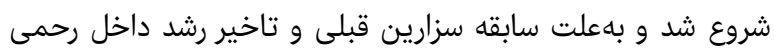

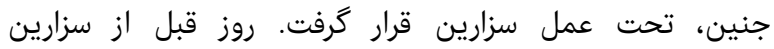

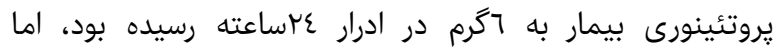

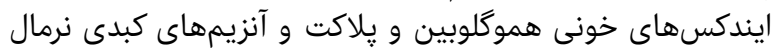

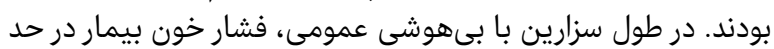

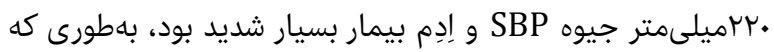

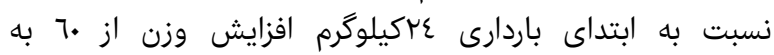

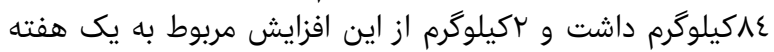

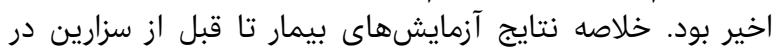
جدول آورآه شده است.

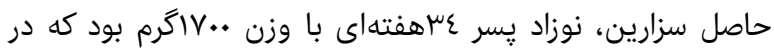

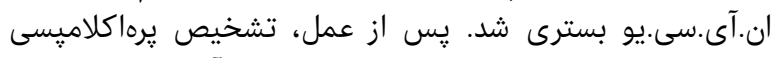

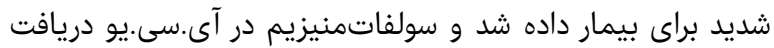

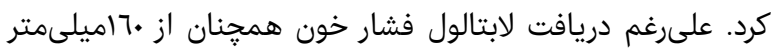

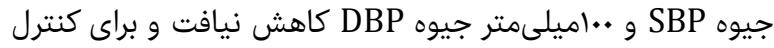

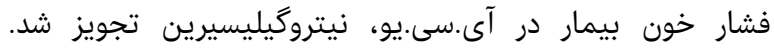

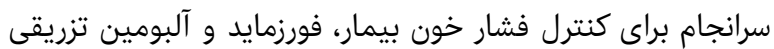

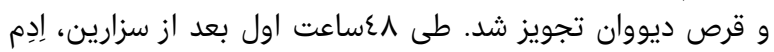

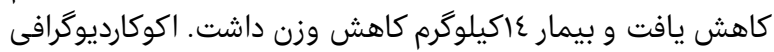

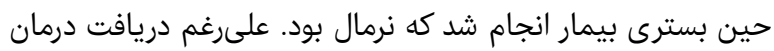

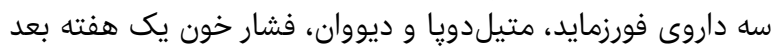


مشاهده مى شد؛ هر حند ممكن بود به دليل تشابه علايم، عارضه

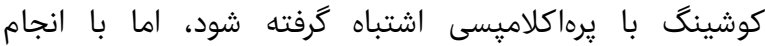

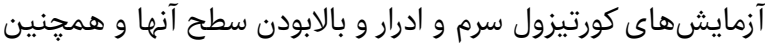

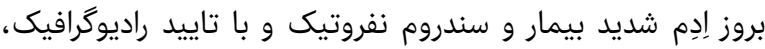

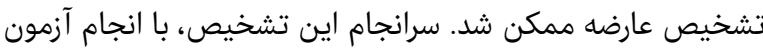

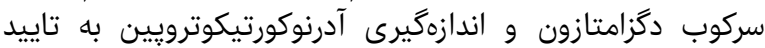

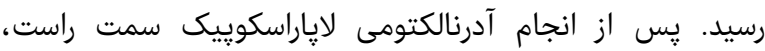

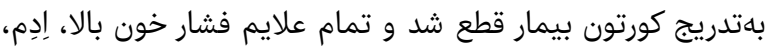

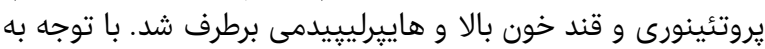

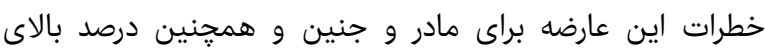

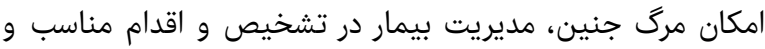
بلهموقع، در نجات مادر و جنين موثر بود.

نتيجه نيرى در نمونهاى كه در اين يزوهش بره بررسى شد، علايم سندروم كوشينگ

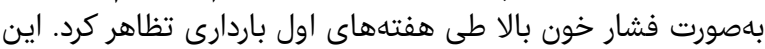

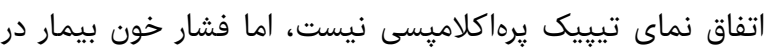

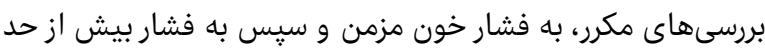

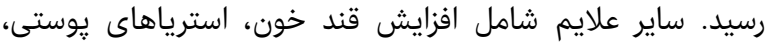

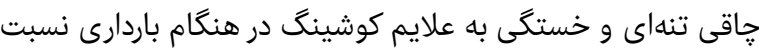

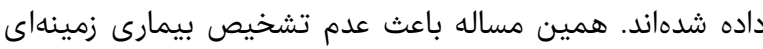

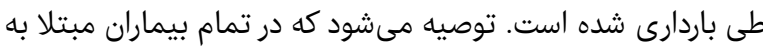

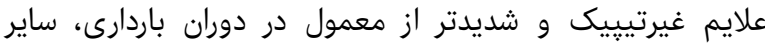

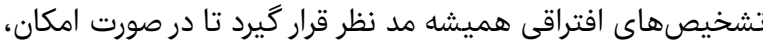

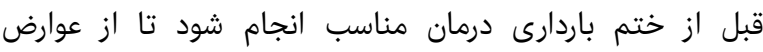
ييشغيرى نمايد.

تشكر و قدردانى: موردى از سوى نويسندگان ذكر نشده است.

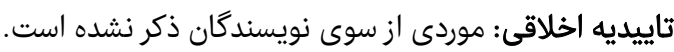

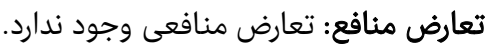
منابع مالى: مركز تحقيقات بارورى ونعارض منافعي وجود نابارورى صاردم منابع مالى اين

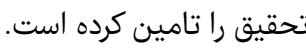

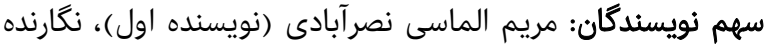

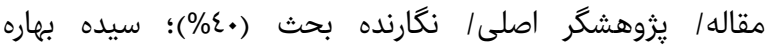

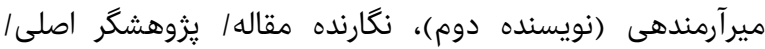

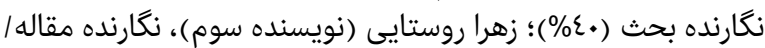

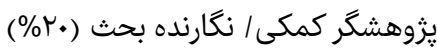

منابع

1- Eschler DC, Kogekar N, Pessah-Pollack R. Management of adrenal tumors in pregnancy. Endocrinol Metab Clin North Am. 2015;44(2):381-97.

2- Katulski K, Podfigurna-Stopa A, Maciejewska-Jeske M, Ruchala M, Gurgul E, Szymankiewicz M, et al. Cushing's syndrome in pregnancy: A case report and mini review of the literature. Gynecol Endocrinol. 2014;30(5):345-9. 3- Sammour RN, Saiegh L, Matter I, Gonen R, Shechner C, Cohen $\mathrm{M}$, et al. Adrenalectomy for adrenocortical adenoma causing Cushing's syndrome in pregnancy: A case report and review of literature. Eur J Obstet Gynecol Reprod Biol. 2012;165(1):1-7.

4- Lekarev 0, New MI. Adrenal disease in pregnancy. Best Pract Res Clin Endocrinol Metab. 2011;25(6):95973.
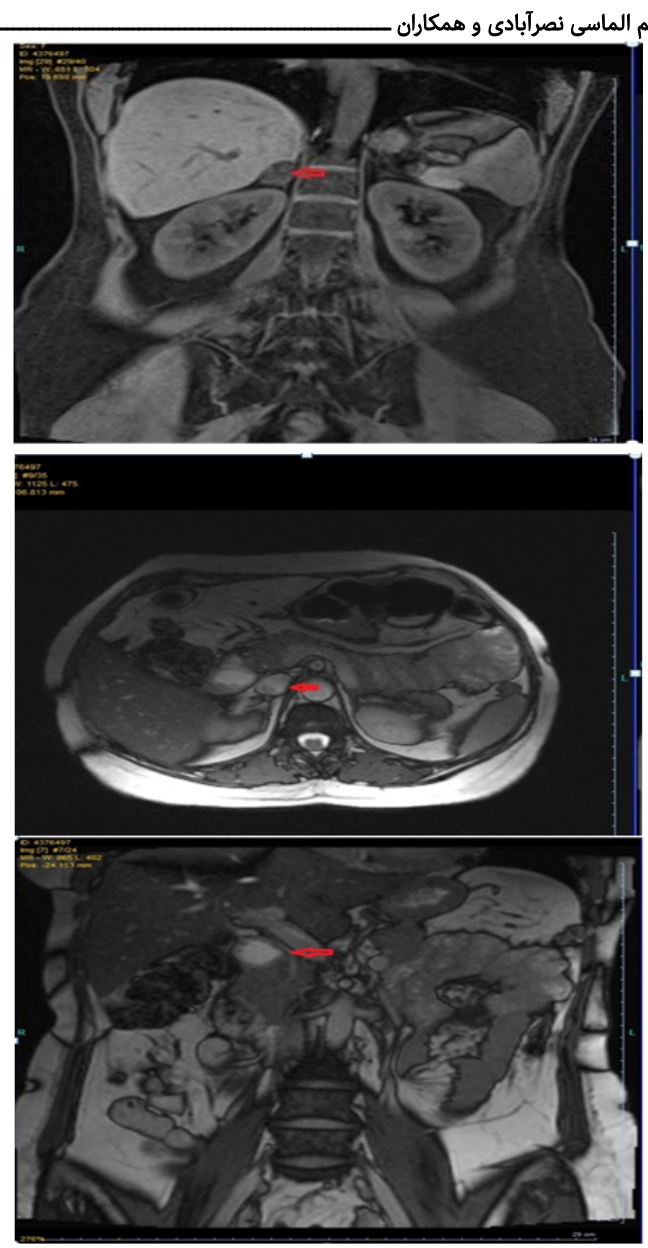

شكل ا) نتيجه MRI بيمار كه بيانكر وجود توده در آدرنال سمت راست است.

بحث

اگرجه سندروم كوشينگ اغلب در سنين بارورى رخ مور مىدهد، اما

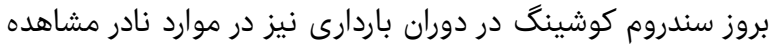

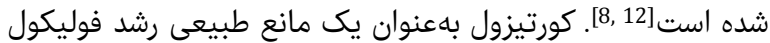

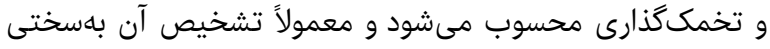

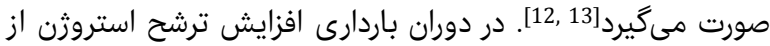

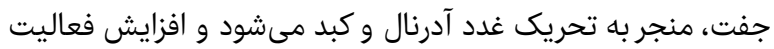

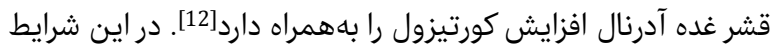

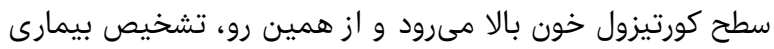

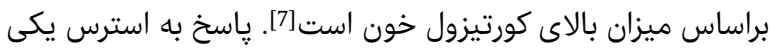

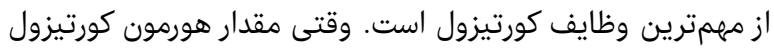

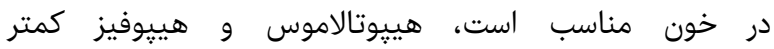

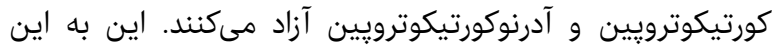

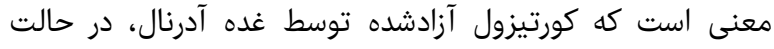

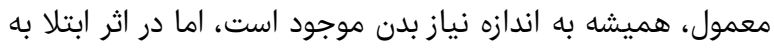

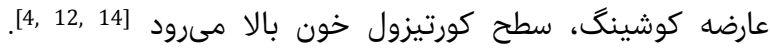

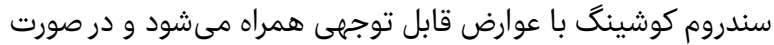

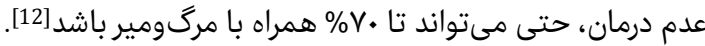

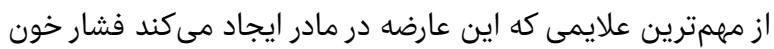

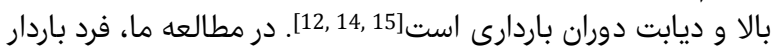

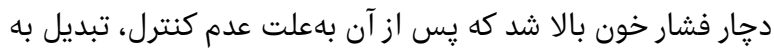

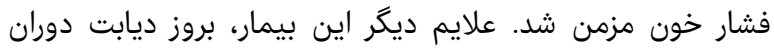

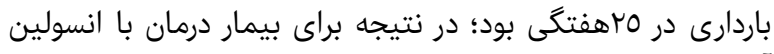

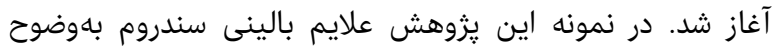


ابتلا به سندروم كوشينگ در يك خانم باردار؛ گزارش موردى 1916

Eur J Obstet Gynecol Reprod Biol. 2013;168(1):1-6

11- Esteghamati A, Eshtiaghi R, Yousefizadeh A,

Nakhjavani M. Diagnosis and management of 253 Cases with Cushing's syndrome in Imam Khomeini Hospital. Tehran Univ Med J. 2007;65(7):77-82.

12- Vilar L, Freitas MdC, Lima LHC, Lyra R, Kater CE. Cushing's syndrome in pregnancy: An overview. Arq Bras Endocrinol Metabol. 2007;51(8):1293-302.

13- Phoon J, Kanalingam D, Chua HL. Adrenal tumours in pregnancy: Diagnostic challenge and management dilemma. Singapore Med J. 2013;54(7):e141-5.

14- Bronstein M, Machado MC, Fragoso MCBV. Management of endocrine disease: Management of pregnant patients with Cushing's syndrome. Eur J Endocrinol. 2015;173(2):R85-91.

15- Aron DC, Schnall AM, Sheeler LR. Cushing's syndrome and pregnancy. Am J Obstet Gynecol. 1990;162(1):244-52.
5- Borna S, Akbari S, Eftekhar T, Mostaan F. Cushing's syndrome during pregnancy secondary to adrenal adenoma. Acta Med Iran. 2012;50(1):76.

6- Nassi R, Ladu C, Vezzosi C, Mannelli M. Cushing's syndrome in pregnancy. Gynecol Endocrinol. 2015;31(2):102-4.

7- Kita M, Sakalidou M, Saratzis A, Ioannis S, Avramides A. Cushing's syndrome in pregnancy: Report of a case and review of the literature. Horm. 2007;6(3):242-6.

8- Achong N, D'EMDEN M, Fagermo N, Mortimer R. Pregnancy-induced Cushing's syndrome in recurrent pregnancies: Case report and literature review. Aust N Z J Obstet Gynaecol. 2012;52(1):96-100.

9- Abdelmannan D, Aron DC. Adrenal disorders in pregnancy. Endocrinol Metab Clin North Am. 2011;40(4):779-94.

10- Lim WH, Torpy DJ, Jeffries WS. The medical management of Cushing's syndrome during pregnancy. 